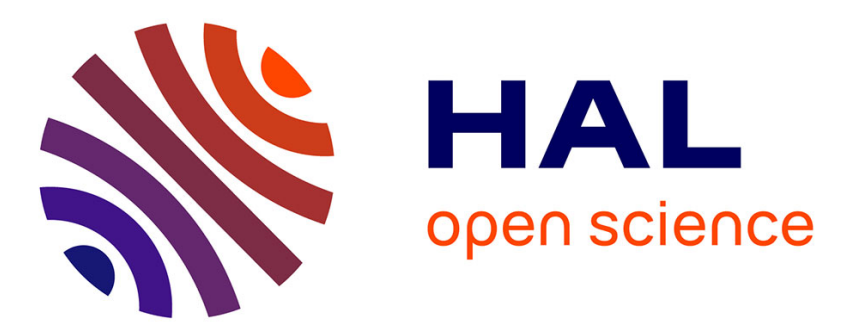

\title{
A Novel Threshold based Compressed Channel Sensing in OFDM System
}

\author{
Hui Xie, Guillaume Andrieux, Yide Wang, Suili Feng, Zhiwen Yu
}

\section{To cite this version:}

Hui Xie, Guillaume Andrieux, Yide Wang, Suili Feng, Zhiwen Yu. A Novel Threshold based Compressed Channel Sensing in OFDM System. AEÜ - International Journal of Electronics and Communications / Archiv für Elektronik und Übertragungstechnik, 2017, 77, pp.149-155. 10.1016/j.aeue.2017.05.002 . hal-01518295

\section{HAL Id: hal-01518295 \\ https://hal.science/hal-01518295}

Submitted on 7 Jul 2017

HAL is a multi-disciplinary open access archive for the deposit and dissemination of scientific research documents, whether they are published or not. The documents may come from teaching and research institutions in France or abroad, or from public or private research centers.
L'archive ouverte pluridisciplinaire HAL, est destinée au dépôt et à la diffusion de documents scientifiques de niveau recherche, publiés ou non, émanant des établissements d'enseignement et de recherche français ou étrangers, des laboratoires publics ou privés. 


\title{
A Novel Threshold based Compressed Channel Sensing in OFDM System
}

\author{
Hui Xie ${ }^{1}$, Guillaume Andrieux ${ }^{2}$, Yide Wang ${ }^{3}$, Suili Feng ${ }^{4}$ and Zhiwen $\mathrm{Yu}^{5}$ \\ ${ }^{1}$ School of Electronic Engineering, Tianjin University of Technology and Education, \\ No.1310, Dagu South Road, Hexi District, Tianjin, China, 300222 \\ ${ }^{2,3}$ Institut d'Electronique et de Télécommunications de Rennes (IETR), UMR 6164 \\ Université Bretagne Loire, Université de Nantes \\ Polytech Nantes, Rue C. Pauc, BP 50609, 44306 Nantes cedex 3, France \\ ${ }^{4}$ School of Electronic and Information Engineering, ${ }^{5}$ School of Computer Science and \\ Engineering, South China University of Technology, \\ ${ }^{4}$ Wushan Road, Tianhe District, Guangzhou, China, 510641 \\ ${ }^{5}$ B3 building, High Education Megacenter, Guangzhou, China, 510006 \\ E-mail: ${ }^{1}$ hui_xie_acad1981@163.com; ${ }^{2}$ guillaume.andrieux@univ-nantes.fr; \\ ${ }^{3}$ yide.wang@univ-nantes.fr ${ }^{4}$ fengsl@scut.edu.cn and ${ }^{5}$ zhwyu@scut.edu.cn
}

\begin{abstract}
High speed data transmission for wireless communication in orthogonal frequency division multiplexing (OFDM) system requires effective channel state information (CSI). CSI should be precisely estimated with low consumption of spectral resources and acceptable computational cost. To realize this goal, an effective compressed sensing (CS) based channel estimation scheme is proposed for sparse channels with large delay spreads, without prior knowledge of channel statistics and noise standard deviation. By fully considering the rank of the measurement matrix, a novel algorithm based on orthogonal matching pursuit (OMP) and least squares (LS) methods with a new threshold is proposed for effective channel estimation. Simulation results show that with fewer number of pilots, the proposed method outperforms the compared existing channel estimation methods in a comprehensive way and approaches
\end{abstract}


the optimal channel estimation performance.

Keywords: Channel estimation, compressed sensing, sparse channels with large delay spreads, orthogonal matching pursuit, OFDM, threshold.

\section{Introduction}

Orthogonal frequency division multiplexing (OFDM) technique is widely used in wireless communication system thanks to its advantages of high data transmission rate over multipath fading channel [1]. In OFDM system, accurate channel state information (CSI), high spectral efficiency, low complexity are all essential. Therefore, how to effectively balance the above factors is one of the main challenges in channel estimation field.

In order to achieve the above goals, the sparse properties of physical channels can be exploited. The existence of physical sparse channels is proven by many experiments [2], which showed that digital television channels [3], underwater acoustic channels [4], some particular urban channels for long term evolution system [5], Hilly Terrain channels [6] and ITU-R vehicular channels (channel A and channel B) [7] have sparse properties.

Traditional channel estimation methods are mainly based on least squares (LS) estimator. Due to the main drawback of LS, which tends to be highly affected by noise, especially in sparse channel situation, the most significant taps (MST) based technique is developed [3]. For MST, different thresholds have been proposed for effective sparse channel estimation. There are generally two types of methods, the first type requires the prior knowledge of channel statistics (power profile of channel impulse response (CIR) or sparsity level) [8, 9], while the second one relies on the estimated noise power or 
noise standard deviation (STD) [10, 11]. [12] shows that threshold without prior requirement of channel statistics will benefit wireless communication system. A two-step threshold is proposed in [11], which realizes effective sparse channel estimation within a wide range of channel sparsity without prior knowledge of both channel statistics and noise STD.

The above mentioned methods assume that $M \geq L_{c p}\left(M\right.$ and $L_{c p}$ are the number of pilots and length of cyclic prefix respectively), which leads to a significant loss on frequency resources in estimating sparse channels with large delay spreads. Therefore, the case of $M<L_{c p}$ becomes more and more popular [13]. The case of $M<L_{c p}$ is closely related to the compressed sensing (CS) [14] theory. $l_{1}$ norm based methods and greedy pursuit methods are two main categories of CS reconstruction methods [4]. Compared with $l_{1}$ norm based methods, greedy pursuit methods have lower complexity, therefore, they are widely used in sparse channel estimation [4, 15, 16, 17]. Recently, orthogonal matching pursuit (OMP), one of the most popular algorithms among greedy pursuit methods and some of its derived algorithms, such as subspace pursuit (SP), adaptive simultaneous orthogonal matching pursuit (A-SOMP) and distributed sparsity adaptive matching pursuit (DSAMP) etc are used to realize channel estimation with prior knowledge of channel statistics (channel sparsity) or signal to noise ratio (SNR) or predetermined threshold [4, 15, 16, 17]. For example, in time domain synchronized OFDM (TDS-OFDM) system, SNR as a prior knowledge is used to estimate the channel sparsity $S$ (number of non-zero channel taps) to improve the SP based sparse channel estimation [16]. In [17], by exploiting the spatially common sparsity within the system bandwidth of multicarrier massive MIMO chan- 
nels during multiple time blocks, the authors proposed distributed sparsity adaptive matching pursuit (DSAMP) algorithm to estimate the downlink channels. Of course, $S_{\max }$ (maximum possible value of $S$ ) can be used [18], however, it may cause loss in estimation precision. We initially proposed an effective threshold estimator for CS based sparse channel estimation without optimizing the noise STD estimation [19], selection of the $m(m \leq M)$ columns in measurement matrix and error vector construction etc.

In this paper, we propose a novel threshold for OMP algorithm without prior knowledge of channel statistics and noise STD. The proposed threshold is based on a novel noise STD estimator, which is realized by a new error vector constructed by $m$ selected coefficients estimated by LS and initial CIR with $S_{\max }$ coefficients estimated by OMP which is different from our initial research work in [19]. The proposed method allows estimating the real number of significant taps $S$. Therefore, the error from the $S_{\max }-S$ noise coefficients can be eliminated. Simulation results show the effectiveness of the proposed method.

This paper is organized as follows. The considered OFDM system model is given in section 2. In section 3, the proposed threshold based estimation algorithm for OMP is presented. Section 4 provides some simulation results. Conclusion is drawn in section 5 .

\section{System Model}

Consider an OFDM system with $N$ subcarriers, among which $M$ are pilots with positions $k_{0}, k_{1}, \ldots, k_{M-1}$. After the transmission of normalized pilots $\boldsymbol{X}_{p}=\operatorname{diag}\left[x\left[k_{0}\right], x\left[k_{1}\right], \ldots, x\left[k_{M-1}\right]\right]$ through a $S$ sparse channel $\boldsymbol{h}=$ 
$\left[h[0], h[1], \ldots, h\left[L_{c p}-1\right]\right]^{T}$, the pilot vector $\boldsymbol{y}_{p}=\left[y\left[k_{0}\right], y\left[k_{1}\right], \ldots, y\left[k_{M-1}\right]\right]^{T}$ is received at the receiver, and given by [2]:

$$
\boldsymbol{y}_{p}=\boldsymbol{A} \boldsymbol{h}+\boldsymbol{w}_{p}
$$

with $\boldsymbol{A}=\boldsymbol{X}_{p} \boldsymbol{F}_{M \times L_{c p}}$ the measurement matrix; $\boldsymbol{F}_{M \times L_{c p}}\left(k_{u}, v\right)=e^{-j\left(2 \pi k_{u} v / N\right)}$, $0 \leq u \leq M-1,0 \leq v \leq L_{c p}-1 ; \boldsymbol{w}_{p}=\left[w\left[k_{0}\right], w\left[k_{1}\right], \ldots, w\left[k_{M-1}\right]\right]^{T}$ the complex additive white Gaussian noise (AWGN) vector with zero mean and covariance matrix $\sigma_{w}^{2} \boldsymbol{I}_{M}$.

\section{Proposed Threshold based Sparse Channel Estimation}

\subsection{Construction of Error Vector with $m$ Selected Columns}

In the case of $M<L_{c p}$, we have $\operatorname{Rank}(\boldsymbol{A})<L_{c p}$. To obtain an effective threshold, the noise STD is required. However, due to $\operatorname{Rank}(\boldsymbol{A})=M$, reconstruct the whole CIR with length $L_{c p}$ and estimate the noise STD are impossible. In this case, partial CIR with $m(m \leq M)$ coefficients can be extracted to estimate the noise STD.

(1) can be rewritten as:

$$
\boldsymbol{y}_{p}=\boldsymbol{A}_{m} \boldsymbol{h}_{m}+\boldsymbol{A}_{b} \boldsymbol{h}_{b}+\boldsymbol{w}_{p}
$$

where $\boldsymbol{A}_{m}=\left[\boldsymbol{a}\left[p_{0}\right], \boldsymbol{a}\left[p_{1}\right], \ldots, \boldsymbol{a}\left[p_{m-1}\right]\right]$ and $\boldsymbol{h}_{m}=\left[h\left[p_{0}\right], h\left[p_{1}\right], \ldots, h\left[p_{m-1}\right]\right]^{T}$ are the matrix with the $m$ selected columns of $\boldsymbol{A}$, which is used to realize the Fourier transform of the partial CIR and vector with the $m$ corresponding channel taps of $\boldsymbol{h}$, which contains the partial information of CIR respectively; $\boldsymbol{A}_{b}$ and $\boldsymbol{h}_{b}=\left[h\left[p_{m}\right], h\left[p_{m+1}\right], \ldots, h\left[p_{L_{c p}-1}\right]\right]^{T}$ are the matrix with the remaining $L_{c p}-m$ columns of $\boldsymbol{A}$ and vector with the corresponding channel taps of $\boldsymbol{h}$ respectively. 
Using LS to estimate the $m$ selected channel taps, we get:

$$
\hat{\boldsymbol{h}}_{l s \_m}=\left(\boldsymbol{A}_{m}^{H} \boldsymbol{A}_{\boldsymbol{m}}\right)^{-1} \boldsymbol{A}_{m}^{H} \boldsymbol{y}_{p}
$$

Combining (2) and (3), we have:

$$
\hat{\boldsymbol{h}}_{l s_{-} m}=\boldsymbol{h}_{m}+\left(\boldsymbol{A}_{m}^{H} \boldsymbol{A}_{m}\right)^{-1} \boldsymbol{A}_{m}^{H} \boldsymbol{A}_{b} \boldsymbol{h}_{b}+\left(\boldsymbol{A}_{m}^{H} \boldsymbol{A}_{m}\right)^{-1} \boldsymbol{A}_{m}^{H} \boldsymbol{w}_{p}
$$

which is composed of three parts, the first part is the $m$ selected channel taps, the second one is the interference due to the significant taps in $\boldsymbol{h}_{b}$ and the last part is the noise part denoted by $\boldsymbol{e}_{m}$.

In order to obtain a good estimate of the error vector $\boldsymbol{e}_{m}$ for effective noise STD estimation, two aspects are essential. The first one is the selection of the number of $m$ and the $m$ columns, which will be discussed in Section 4 and Section 3.1.1 respectively, the second one is the reduction of the interference from the first two parts in (4), which will be discussed in Section 3.1.2.

\subsubsection{Selection of $m$ Columns}

The condition number of $\boldsymbol{A}_{m}$ is a key parameter for LS method. The smaller it is, the better is the stability of the estimator to perturbations [20].

To obtain the $m$ effective selected columns, $\operatorname{cond}\left(\boldsymbol{A}_{m}\right)$ should be the smallest possible. To realize this, the subset with $m$ indices should be chosen as:

$$
\Lambda=\arg \min _{\Lambda_{1}} \operatorname{cond}\left(\boldsymbol{A}_{\Lambda_{1}}\right),\left|\Lambda_{1}\right|=m
$$

with $\Lambda$ the subset of the $m$ optimally selected columns. The optimal partial measurement matrix denoted by $\boldsymbol{A}_{m}$ is obtained by choosing the $m$ columns defined by $\Lambda$. 
(5) is the optimal solution for the estimation of $\boldsymbol{h}_{m}$, however, to obtain $\Lambda$, there are $\left(\begin{array}{c}L_{c p} \\ m\end{array}\right)$ possible choices. It is a heavy computational task. Similar to the suboptimal pilot arrangement method proposed in [21], a suboptimal subset of indices of $m$ selected columns can be obtained. Firstly, a limited number of subsets of $m$ selected columns are randomly generated, then, the suboptimal subset is given by the matrix with the smallest condition number.

\subsubsection{Reduction of Interference and Construction of Error Vector}

In order to get a good estimate $\hat{\boldsymbol{e}}_{m}$, it is necessary to reduce the impact of the significant taps of $\boldsymbol{h}_{m}$ and $\boldsymbol{h}_{b}$ in (4). Therefore, the significant taps estimated by OMP expressed by $\hat{h}_{\text {omp }}\left[d_{0}\right], \hat{h}_{\text {omp }}\left[d_{1}\right], \ldots, \hat{h}_{\text {omp }}\left[d_{S_{\max }-1}\right], 0 \leq$ $d_{0}, d_{1}, \ldots, d_{S_{\max }-1} \leq L_{c p}-1$ are introduced. The significant taps of $\hat{\boldsymbol{h}}_{o m p}$ in subsets $\Lambda$ and $\Lambda^{c}$ (the complement of $\Lambda$ ) are extracted to get two new sparse vectors $\hat{\boldsymbol{h}}_{\text {omp_m }}$ and $\hat{\boldsymbol{h}}_{\text {omp_b }}$. Then, a new equation is obtained with regard to $\hat{\boldsymbol{e}}_{m}$ :

$$
\begin{aligned}
\hat{\boldsymbol{e}}_{m} & =\boldsymbol{h}_{m}-\hat{\boldsymbol{h}}_{\text {omp_m }}+\left(\boldsymbol{A}_{m}^{H} \boldsymbol{A}_{m}\right)^{-1} \boldsymbol{A}_{m}^{H} \boldsymbol{A}_{b}\left(\boldsymbol{h}_{b}-\hat{\boldsymbol{h}}_{o m p \_b}\right)+\left(\boldsymbol{A}_{m}^{H} \boldsymbol{A}_{m}\right)^{-1} \boldsymbol{A}_{m}^{H} \boldsymbol{w}_{p} \\
& =\hat{\boldsymbol{h}}_{l s \_m}-\hat{\boldsymbol{h}}_{o m p \_m}-\left(\boldsymbol{A}_{m}^{H} \boldsymbol{A}_{m}\right)^{-1} \boldsymbol{A}_{m}^{H} \boldsymbol{A}_{b} \hat{\boldsymbol{h}}_{\text {omp_ } b}
\end{aligned}
$$

From (4) and (6), $\hat{\boldsymbol{e}}_{m}$ can finally be rewritten as:

$$
\hat{\boldsymbol{e}}_{m}=\hat{\boldsymbol{h}}_{l s \__{-} m}-\left[\begin{array}{ll}
\boldsymbol{I}_{m} & \left(\boldsymbol{A}_{m}^{H} \boldsymbol{A}_{m}\right)^{-1} \boldsymbol{A}_{m}^{H} \boldsymbol{A}_{b}
\end{array}\right]\left[\begin{array}{c}
\hat{\boldsymbol{h}}_{\text {omp_m }} \\
\hat{\boldsymbol{h}}_{\text {omp_b }}
\end{array}\right]
$$

\subsection{Noise STD and Threshold Estimation}

If the initial estimated channel by OMP is accurate, $\hat{\boldsymbol{e}}_{m}$ approximates the noise vector $\boldsymbol{e}_{m}=\left(\boldsymbol{A}_{m}^{H} \boldsymbol{A}_{m}\right)^{-1} \boldsymbol{A}_{m}^{H} \boldsymbol{w}_{p}$ with length of $m$. Each element of 
$\boldsymbol{e}_{m}$ being a linear combination of independent complex Gaussian variables, is a complex Gaussian random variable. In practice, some small contribution of significant taps remains in $\hat{\boldsymbol{e}}_{m}$, it is better to use the median absolute deviation (MAD) method $[11,22]$. In the following, MAD method is adopted to realize effective noise STD estimation.

Each element of $\boldsymbol{e}_{m}$ follows complex Gaussian distribution. The STD of its real part $\hat{\sigma}_{r}$ and imaginary part $\hat{\sigma}_{i}$ is estimated by the median value [11]:

$$
\hat{\sigma}_{r}=\hat{\sigma}_{i}=\hat{\sigma}^{\prime}=\frac{\operatorname{median}\left(\left|\hat{\boldsymbol{e}}_{m}\right|\right)}{\sqrt{\ln 4}}
$$

Therefore, the STD of each element of $\boldsymbol{e}_{m}$ can be estimated by $\hat{\sigma}=$ $\sqrt{2} \hat{\sigma}^{\prime}$. With the estimated $\hat{\sigma}$, the universal threshold can be adopted for the detection of sparse channel [2, 11, 22]:

$$
\hat{T}=R \hat{\sigma} ;\left(R=\sqrt{2(1+a) \ln \left(L_{c p}\right)}\right)
$$

with $a,(a \geq 0)$ a constant factor. In this paper, we take $a=0$.

\subsection{Main Framework of the Proposed Method}

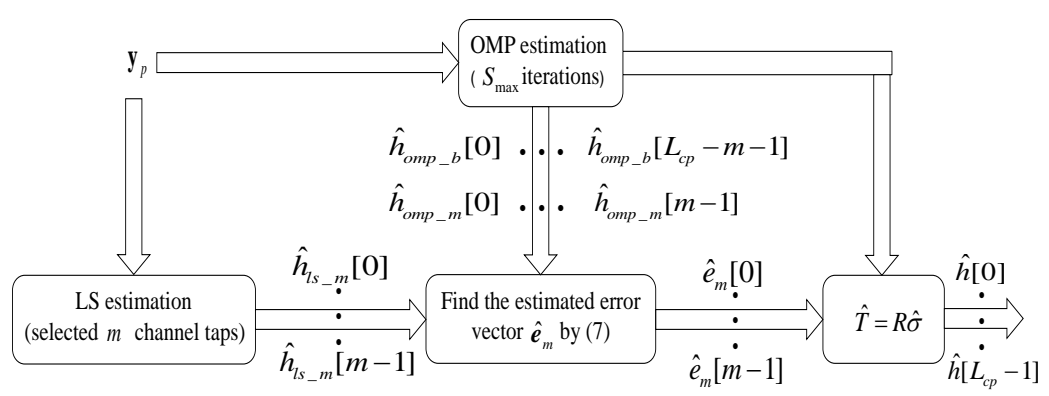

Figure 1: Proposed channel estimation method

Fig 1 gives the main framework of the proposed method, the specific steps of which are summarized as follows. 
Step 1: Get an initial CIR with $S_{\max }$ iterations by OMP algorithm.

Step 2: Estimate the partial CIR with $\boldsymbol{y}_{p}$ by LS (3).

Step 3: Obtain the estimated error vector $\hat{\boldsymbol{e}}_{m}$ by $(7)$.

Step 4: Estimate the noise STD $\hat{\sigma}$ from (8) and the threshold $\hat{T}$ by (9).

Step 5: Detect the MST by:

$$
\hat{h}[n]=\left\{\begin{array}{cl}
\hat{h}_{o m p}[n], & \left|\hat{h}_{o m p}[n]\right|>\hat{T} \\
0, & \left|\hat{h}_{o m p}[n]\right| \leq \hat{T}
\end{array} \quad, 0 \leq n \leq L_{c p}-1\right.
$$

\section{Simulation Results}

In simulation, a QPSK modulated OFDM system with length of cyclic prefix 256, is considered. The system has a total bandwidth of $10 \mathrm{MHz}$ [9] and 1024 subcarriers, among which minimum 128 are pilots. For obtaining a comprehensive performance evaluation of different channel estimation methods, two different sparse channel models with different channel sparsities and channel statistics are considered. The first channel model is the ATTC (Advanced Television Technology Center) and the Grand Alliance DTV laboratory's ensemble E model whose CIR is given by [3]:

$h[n]=\delta[n]+0.3162 \delta[n-2]+0.1995 \delta[n-17]+0.1296 \delta[n-36]+0.1 \delta[n-75]+0.1 \delta[n-137]$.

where the unit delay of the channel is equal to the OFDM sample period.

The second channel model is the Hilly Terrain channel [6] whose power delay profile (POD) is given in Table 1.

The coefficients in (11) and the square root of the elements of the relative power in their linearized form in Table 1 represent the STD of the corresponding zero mean complex Gaussian random variables. Additionally, the 
Table 1: Power delay profile for 12 tap Hilly Terrain channel

\begin{tabular}{|c|c|c|c|c|c|c|c|c|c|c|c|c|}
\hline Delay $[\mu s]$ & 0 & 0.2 & 0.4 & 0.6 & 0.8 & 2.0 & 2.4 & 15 & 15.2 & 15.8 & 17.2 & 20 \\
\hline Power $[\mathrm{dB}]$ & -10 & -8 & -6 & -4 & 0 & 0 & -4 & -8 & -9 & -10 & -12 & -14 \\
\hline
\end{tabular}

pilot pattern arrangement is obtained by the suboptimal method proposed in [21]. Moreover, we consider $S_{\max }=20$ and 6000 randomly generated subsets of indices are used for searching a suboptimal subset of indices selection of $m$ selected columns.

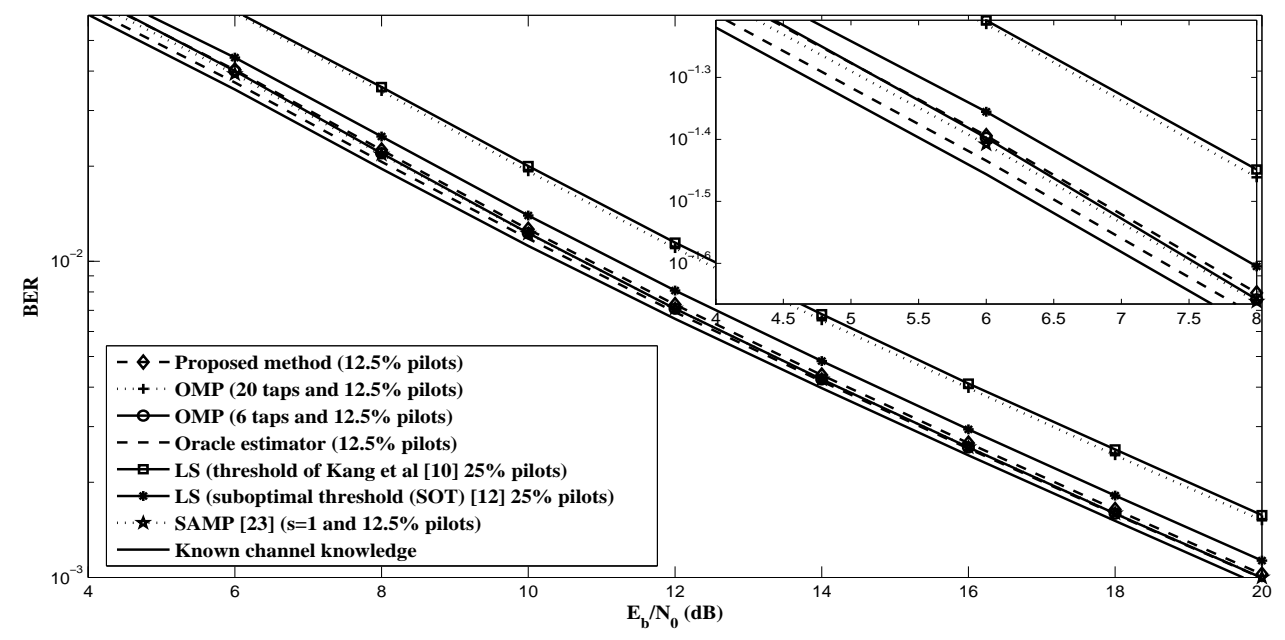

Figure 2: BER performance comparison for the first channel model

Fig 2 illustrates BER performance comparison of different channel estimation methods for the first channel model. We observe that even with only $12.5 \%$ of pilots, the proposed method significantly outperforms the LS method with the threshold proposed by Kang et al. [10] (for convenient comparisons, the exact noise STD is used) with $25 \%$ of pilots and OMP method with 20 taps and $12.5 \%$ of pilots throughout the considered $\mathrm{E}_{\mathrm{b}} / \mathrm{N}_{0}$; the $\mathrm{E}_{\mathrm{b}} / \mathrm{N}_{0}$ gap is about $2 \mathrm{~dB}$ for the same BER performance. Additionally, 
the proposed method slightly outperforms the LS estimator with sub-optimal threshold (SOT) [12] (the estimated number of channel taps $\hat{S}$ is set to be the channel sparsity $S$ ) and $25 \%$ of pilots in the overall considered $\mathrm{E}_{\mathrm{b}} / \mathrm{N}_{0}$, however, the spectral efficiency of the proposed method is much better. Furthermore, without prior knowledge of either channel statistics and noise STD, the proposed method still maintains good performance on BER compared with sparsity adaptive matching pursuit (SAMP) method (step size $s=1$ ) [23] with the prior knowledge of noise STD and 12.5\% of pilots, OMP method with 6 taps (exact sparsity $S$ in the first channel model), the oracle estimator (constrained LS with known number of non-zero channel coefficients and their positions) [24] and known channel CSI (known instantaneous channel frequency response). When compared with SAMP method, the oracle estimator with $12.5 \%$ of pilots and known CSI, the proposed method has at most $0.15 \mathrm{~dB}, 0.5 \mathrm{~dB}$ and $0.8 \mathrm{~dB}$ performance degradation respectively.

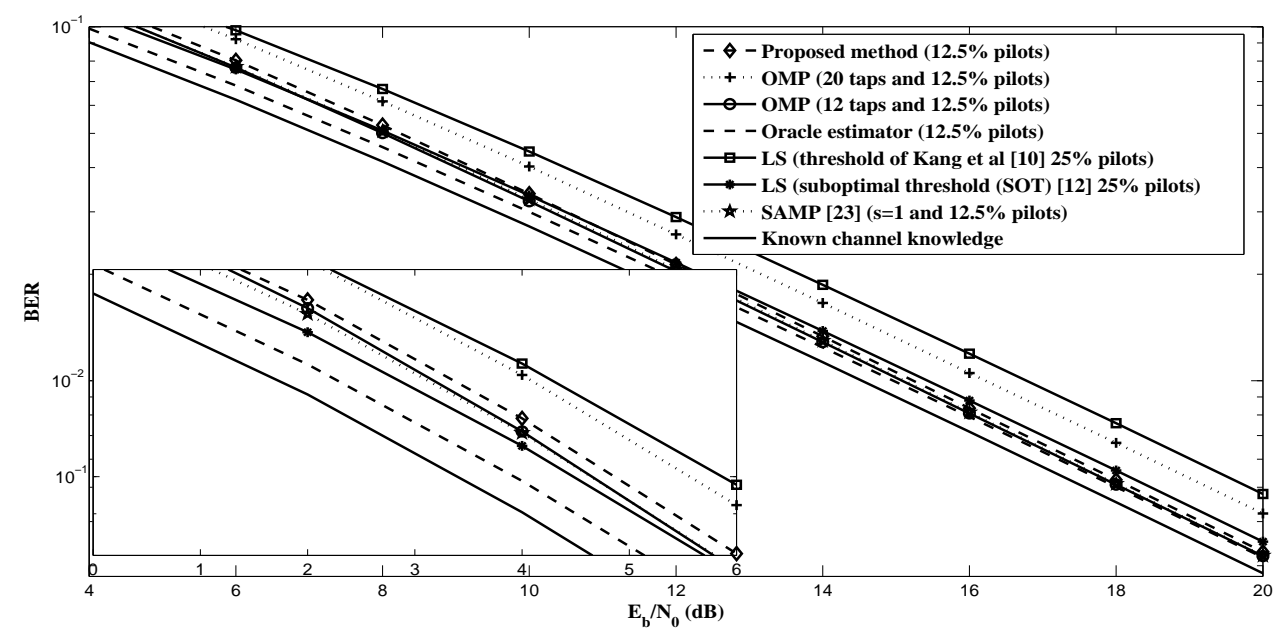

Figure 3: BER performance comparison for the second channel model

As shown in Fig 3, compared with the first channel model, although the 
second channel model has different channel sparsity, delay of channel paths and POD, the proposed method still maintains similar trends in BER performance compared with other channel estimation methods except that the LS estimator with sub-optimal threshold (SOT) and 25\% of pilots outperforms slightly the proposed method with $12.5 \%$ of pilots (at most $0.6 \mathrm{~dB}$ degradation in $\mathrm{E}_{\mathrm{b}} / \mathrm{N}_{0}$ for the same BER) in low $\mathrm{E}_{\mathrm{b}} / \mathrm{N}_{0}$ (from $0 \mathrm{~dB}$ to $10 \mathrm{~dB}$ ). The main reasons are the reduction of number of pilots and the increase of channel sparsity, which lead to the decrease on the performance of sparse channel reconstruction by OMP algorithm in low $\mathrm{E}_{\mathrm{b}} / \mathrm{N}_{0}$. However, considering the spectral efficiency promotion, the precision loss is acceptable.

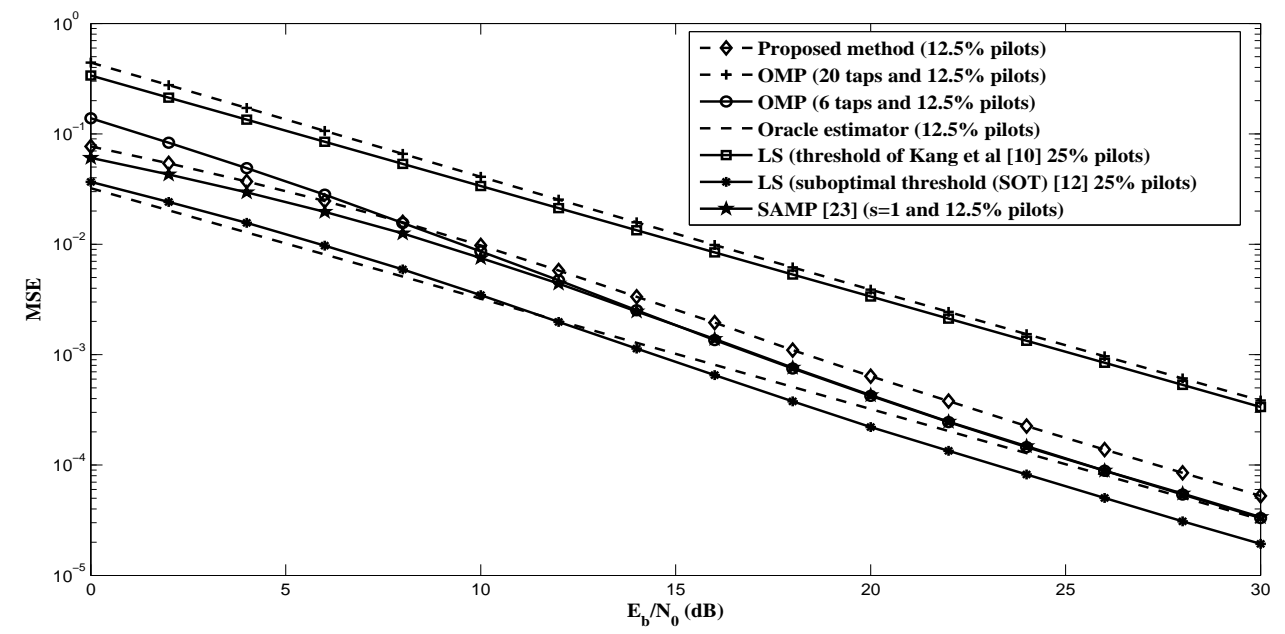

Figure 4: MSE performance comparison for the first channel model

In Fig 4, MSE performance of different channel estimation methods is evaluated for the first channel model. In vast majority of considered $E_{b} / N_{0}$ range (from $12 \mathrm{~dB}$ to $30 \mathrm{~dB}$ ), the LS estimator with SOT and $25 \%$ of pilots outperforms the methods with $12.5 \%$ of pilots, including, oracle estimator, OMP method with 6 taps, SAMP method and the proposed method, which 
doesn't coincide with the BER performance in Fig 2. The primary reason for this is that MSE and BER are expressed in terms of $E_{b} / N_{0}$ (instead of SNR), which is more fair for the channel estimation methods with different pilot percentages. In this paper, $12.5 \%$ and $25 \%$ of pilots are considered, therefore, by considering the parameters of $N=1024, L_{c p}=256$ and QPSK modulation used in the simulation, $\mathrm{SNR}=\mathrm{E}_{\mathrm{b}} / \mathrm{N}_{0}+10 \log \left(n_{b} \frac{N-M}{N} \frac{N}{N+L_{c p}}\right)=$ $\mathrm{E}_{\mathrm{b}} / \mathrm{N}_{0}+1.46 \mathrm{~dB}$ in the case of $12.5 \%$ of pilots (For QPSK modulation, one symbol contains 2 bits, so $n_{b}=2$. Additionally, in the case of $12.5 \%$ of pilots, $M=128$ ), while in the case of $25 \%$ of pilots, $\mathrm{SNR}=\mathrm{E}_{\mathrm{b}} / \mathrm{N}_{0}+0.79 \mathrm{~dB}$ (The same calculation formula, but in the case of $25 \%$ of pilots, $M=256$ ), the power payload of the methods with $25 \%$ of pilots is $0.67 \mathrm{~dB}$ higher than that of the methods with $12.5 \%$ of pilots. If we consider the LS estimator with SOT and $25 \%$ of pilots in $6 \mathrm{~dB}$ and the oracle estimator with $12.5 \%$ of pilots in $5.33 \mathrm{~dB}$ in the small figure in Fig 2, their BER performance is the same and $\mathrm{E}_{\mathrm{b}} / \mathrm{N}_{0}$ gap is about $0.67 \mathrm{~dB}$, which actually corresponds approximately to the power payload gap. Additionally, considering the same channel estimation performance, the $\mathrm{E}_{\mathrm{b}} / \mathrm{N}_{0}$ gap between the LS estimator with SOT and $25 \%$ of pilots and the oracle estimator with $12.5 \%$ of pilots in Fig 2 is less than $0.6 \mathrm{~dB}$ within the $\mathrm{E}_{\mathrm{b}} / \mathrm{N}_{0}$ range $10 \mathrm{~dB}-20 \mathrm{~dB}$, which can explain why the LS estimator with SOT and $25 \%$ of pilots has better performance in MSE.

The above analysis on the performance of the proposed method considers $m=80$. To show the influence of the value of $m$, in Fig 5 , the BER performance of the proposed method with different $m$ for the first channel model is shown. Generally, the proposed method with $m=20,40,60,80,100$ and $12.5 \%$ of pilots has almost the same BER performance throughout the 


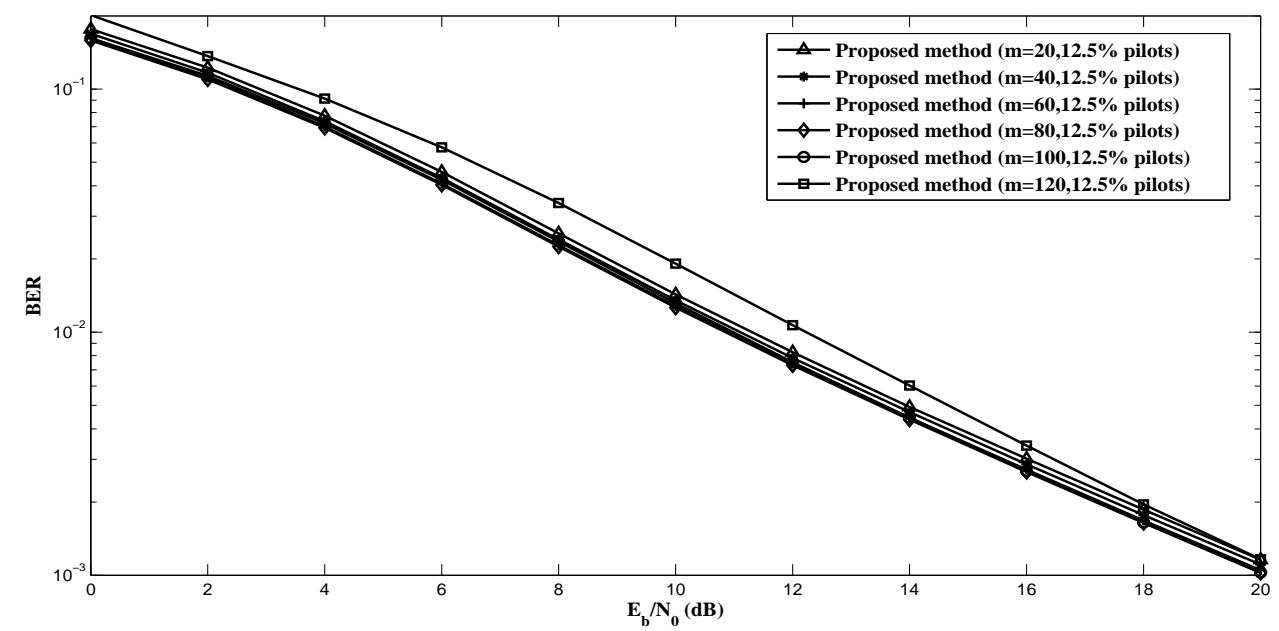

Figure 5: BER performance of proposed methods with different $m$ for the first channel model

considered $\mathrm{E}_{\mathrm{b}} / \mathrm{N}_{0}$. Comparatively, the proposed method with $m=120$ and $12.5 \%$ of pilots has obvious poorer performance. To understand the reason, we consider the absolute relative error on the proposed estimated STD with the first channel model $\varepsilon=\frac{\sum_{i=0}^{U-1}\left|\hat{\sigma}_{i}-\sigma\right|}{U \sigma}\left(\sigma\right.$ is the exact noise STD, $\hat{\sigma}_{i}$ is the proposed estimated STD in the $i^{\text {th }}$ Monte-Carlo simulation and $U$ is the number of Monte Carlo simulations), which is shown in Table 2.

Table 2: Performance of the proposed noise STD estimation method with different $m$ for the first channel model

\begin{tabular}{|c|c|c|c|c|c|c|}
\hline $\mathrm{E}_{\mathrm{b}} / \mathrm{N}_{0}(\mathrm{~dB})$ & $m=20$ & $m=40$ & $m=60$ & $m=80$ & $m=100$ & $m=120$ \\
\hline 10 & 0.24 & 0.19 & 0.11 & 0.11 & 0.37 & 1.64 \\
\hline 20 & 0.24 & 0.18 & 0.11 & 0.11 & 0.38 & 1.64 \\
\hline 30 & 0.23 & 0.18 & 0.11 & 0.11 & 0.38 & 1.64 \\
\hline
\end{tabular}

As can be seen from Table 2, for the same value of $m, \varepsilon$ is extremely stable 
for different $\mathrm{E}_{\mathrm{b}} / \mathrm{N}_{0}$. When $m=60,80, \varepsilon$ is approximately 0.11 . However, when $m=120, \varepsilon$ is 1.64, which is a huge error for noise STD estimation.

Table 3: Condition number of the measurement matrix $\boldsymbol{A}_{m}$

\begin{tabular}{|c|c|c|c|c|c|c|}
\hline$m$ & 20 & 40 & 60 & 80 & 100 & 120 \\
\hline condition number & 1.66 & 2.43 & 3.55 & 5.26 & 9.29 & 24.71 \\
\hline
\end{tabular}

Table 3 presents the condition number of $\boldsymbol{A}_{m}$ with different $m$. When $m=$ 20,40,60,80, the condition number of $\boldsymbol{A}_{m}$ is less than 5.5. Comparatively, when $m=100$, the condition number is 9.29 , which is a bigger number, however, it is not a too big value for condition number, therefore, $\varepsilon$ difference is also limited. However, when $m=120$ close to the number of pilots 128 , the condition number of $\boldsymbol{A}_{m}$ is 24.71 , which is nearly 5 times as large as that for $m=80$. This can explain why the noise STD estimation and BER performance are poor when $m=120$.

From the above analysis combined with the performance of the proposed noise STD estimation method with different $m$ in Table 2 and the condition number of $\boldsymbol{A}_{m}$ in Table 3, we know that the choice of the value of $m$ should effectively balance the number of noise samples $m$ and the condition number of $\boldsymbol{A}_{m}$. In practice, $m / M$ should be considered. In this paper $M=128$. The cases $m=60$ and $m=80$ can achieve the best noise STD performance. Therefore, a good value for $m / M$ should be within the range $[0.47,0.63]$.

Table 4 evaluates the complexity of the proposed method and the traditional DFT based method. As can be seen from the table, the complexity of the proposed method is composed of that of OMP method $O\left(M L_{c p} S_{\max }\right)$, threshold estimation method $O\left(M^{2} m\right)$ and FFT method $O\left(N \log _{2} N\right)$. In the 
Table 4: Computational complexity comparison

\begin{tabular}{|c|c|c|c|c|}
\hline \multirow{2}{*}{ Alg } & \multicolumn{2}{|c|}{ Proposed Method } & \multicolumn{2}{c|}{ LS based method } \\
\cline { 2 - 5 } & $\mathrm{OMP}+\mathrm{Thr}$ & $\mathrm{FFT}$ & $\mathrm{LS}+\mathrm{Thr}$ & $\mathrm{FFT}$ \\
\hline Comp & $O\left(M L_{c p} S_{\max }\right)$ & $O\left(N \log _{2} N\right)$ & $O\left(M \log _{2} M\right)$ & $O\left(N \log _{2} N\right)$ \\
\hline
\end{tabular}

proposed method, the complexity of OMP algorithm is $O\left(M L_{c p} S_{\max }\right)$, which approximates to $O\left(M^{2} m\right)$, therefore, the total complexity of the proposed method is $O\left(M L_{c p} S_{\text {max }}\right)+O\left(N \log _{2} N\right)$. LS based method has complexity of LS estimator, threshold estimation and FFT method. The complexity of LS and threshold methods is $O\left(M \log _{2} M\right)$ and the complexity of FFT method is $O\left(N \log _{2} N\right)$, therefore, the total complexity is $O\left(N \log _{2} N\right)$. Although, the proposed method has a higher complexity than LS based method, the estimation performance and spectral efficiency of the proposed method are much better than LS based method. (Note: The complexity of the algorithm of condition number based $m$ columns selection and that of pilot arrangement is not included, because they can be realized in the absence of practical communication.) SAMP method $(s=1)$ requires a few more iterations than OMP as indicated in [23], so the complexity of SAMP is higher than the proposed method, which is also confirmed by the simulation time. Additionally, SAMP method requires the prior knowledge of noise STD, although its estimation performance is slightly better.

\section{Conclusion}

This paper addresses the CS based sparse channel estimation in OFDM system. The main goal of the paper is to detect MST for CS based sparse 
channel estimation without prior knowledge of channel statistics and noise STD. In the proposed method, by employing jointly LS and OMP, an error vector with $m$ coefficients, which approximates the noise vector, is constructed. By employing the $m$ coefficients of the error vector, the noise STD is estimated and effective MST detection is realized. Simulation results show that the proposed method has the advantages in comprehensive performances regarding the channel estimation performance, spectral efficiency, computational complexity and prior parameters dependence (noise STD).

\section{Acknowledgment}

This work was supported by China Scholarship Council (No. 2010615039), Research Fund of Tianjin University of Technology and Education (No. KRKC 011614 and No. KJ15-16) and Science and Technology Planning Project of Guangdong Province, China (No. 2015A050502011 and No. 2016A010101009).

\section{References}

[1] J. A. C. Bingham.: 'Multicarrier modulation for data transmission: An idea whose time has come', IEEE Commun. Mag., May 1990, 28, (5), pp. $5-14$.

[2] W.U. Bajwa, J. Haupt, A. M. Sayeed, R. Nowak.: 'Compressed Channel Sensing: A new Approach to Estimating Sparse Multipath Channels', Proc. of IEEE., Jun 2010, 98, (6), pp. 1058-1076.

[3] H. Minn, V. K. Bhargava, 'An investigation into time-domain approach for OFDM channel estimation', IEEE Trans. Broadcast., Dec 2000, 46, (4), pp. 240-248. 
[4] C. R. Berger, Z. H. Wang, J. Z. Huang, S. L. Zhou.: 'Application of compressive sensing to sparse channel estimation', IEEE Commun. Mag., Nov 2010, 48, (11), pp. 164-174.

[5] P. Maechler, P. Greisen, B. Sporrer, S. Steiner, N. Felber, A. Burg.: 'Implementation of greedy algorithms for LTE sparse channel estimation', in Proc. 44th Asilomar Conf. Signals, Syst and Comp., Nov 2010. pp. 400-405.

[6] K. M. Nasr, J. P. Cosmas, M. Bard, J. Gledhill.: 'Performance of an echo canceller and channel estimator for on-channel repeaters in DVBT/H networks', IEEE Trans. Broadcast., Sep 2007, 53, (3), pp. 609-618.

[7] Recommendation ITU-R M.1225, International Telecommunication Union, Guidelines for evaluation of radio transmission technologies for IMT-2000. 1997.

[8] J. Oliver, R. Aravind, K. M. M. Prabhu.: 'Sparse channel estimation in OFDM system by threshold-based pruning', Electron. Lett., Jun 2008, 44, (13), pp. 830-832.

[9] L. Najjar.: 'Sparsity level-aware threshold-based channel structure detection in OFDM systems', Electron. Lett., Apr 2012, 48, (9), pp. 495496.

[10] Y. Kang, K. Kim, H. Park.: 'Efficient DFT-based channel estimation for OFDM system on multipath channels', IET Commun., Apr 2007, 1, (2), pp. 197-202. 
[11] H. Xie, G. Andrieux, Y. Wang, J. F. Diouris, S. Feng.: 'Efficient time domain threshold for sparse channel estimation in OFDM system', AEUInternational Journal of Electron. and Commun., Apr 2014, 68, (4), pp. 277-281.

[12] S. Rosati, G. E. Corazza, A. Vanelli-Coralli.: 'OFDM channel estimation based on impulse response decimation: Analysis and novel algorithms', IEEE Trans on Commun., Jul 2012, 60, (7), pp. 1996-2008.

[13] G. Tauböck, F. Hlawatsch.: 'A compressed sensing technique for OFDM channel estimation in mobile environments: Exploiting channel sparsity for reducing pilots', in Proc. IEEE Int. Conf. Acoust., Speech, and Signal Process (ICASSP)., Apr. 2008, pp. 2885-2888.

[14] D. L. Donoho.: 'Compressed Sensing', IEEE Trans. Inf. Theory., Apr 2006, 52, (4), pp. 1289-2306.

[15] L. Dai, J. Wang, Z. Wang, P. Tsiaflakis, and M. Moonen.: 'Time domain synchronous OFDM based on simultaneous multi-channel reconstruction', in Proc. IEEE Int. Commun. Conf (ICC), Jun 2013, pp. 29842989.

[16] X. Zhu, J. Wang, L. Dai, Z. Wang.: 'Sparsity-Aware Adaptive Channel Estimation Based on SNR Detection', IEEE Trans. Broadcast., Mar. 2015, 61, (1), pp. 119-126.

[17] Z. Gao, L. Dai, Z. Wang, S. Chen.: 'Spatially common sparsity based adaptive channel estimation and feedback for FDD massive MIMO', IEEE Trans. Signal Proces., Dec 2015, 63, (23), pp. 6169-6183. 
[18] X. Jiang, W. J. Zeng, E. Cheng.: 'A fast algorithm for sparse channel estimation via orthogonal matching pursuit', in Proc. 73rd Vehicular Technology Conf. (VTC)., May 2011, pp.1-5.

[19] H. Xie, G. Andrieux, Y. Wang, J. F. Diouris, S. Feng.: 'A novel effective compressed sensing based sparse channel estimation in OFDM system', in Proc. IEEE Int Conf. Signal Process, Commun and Comp (ICSPCC)., Aug 2013, pp. 1-6.

[20] O. Mostovyi.: 'On the stability the least squares Monte Carlo', Optim Lett., Feb 2013, 7, (2), pp. 259-265.

[21] X. He, R. Song.: 'Pilot pattern optimization for compressed sensing based sparse channel estimation in OFDM systems', in Proc. Wireless Commun and Signal Process Conf., Oct 2010.

[22] D. L. Donoho, I. M. Johnstone.: 'Ideal spatial adaptation by wavelet shrinkage', Biometrika., 1994, 81, (3), pp. 425-455.

[23] T. T. Do, L. Gan, N. Nguyen, T. D. Tran.: 'Sparsity adaptive matching pursuit algorithm for practical compressed sensing', in Proc. 42nd Asilomar Conf. Signal Syst. Comput. (Pacific Grove, CA), Oct. 2008, pp. 581-587.

[24] Z. Ben-Haim, Y. Eldar, M. Elad: 'Coherence-based performance guarantees for estimating a sparse vector under random noise', IEEE Trans Signal Process., Oct 2010, 58, (10), pp. 5030-5043. 